Brain, Behavior and Evolution
Brain Behav Evol 2018;91:97-108

DOI: $10.1159 / 000488873$
Received: February 13, 2018

Returned for revision: March 13, 2018

Accepted after revision: March 28, 2018

Published online: June 6, 2018

\title{
Adaptations for Substrate Gleaning in Bats: The Pallid Bat as a Case Study
}

\author{
Khaleel A. Razak \\ Department of Psychology and Graduate Neuroscience Program, University of California, Riverside, CA, USA
}

\section{Keywords}

Bats · Foraging · Neuroethology · Stealth echolocation · Venom resistance $\cdot$ Sound localization · Pinna morphology

\begin{abstract}
Substrate gleaning is a foraging strategy in which bats use a mixture of echolocation, prey-generated sounds, and vision to localize and hunt surface-dwelling prey. Many substrategleaning species depend primarily on prey-generated noise to hunt. Use of echolocation is limited to general orientation and obstacle avoidance. This foraging strategy involves a different set of selective pressures on morphology, behavior, and auditory system organization of bats compared to the use of echolocation for both hunting and navigation. Gleaning likely evolved to hunt in cluttered environments and/or as a counterstrategy to reduce detection by eared prey. Gleaning bats simultaneously receive streams of echoes from obstacles and prey-generated noise, and have to segregate these acoustic streams to attend to one or both. Not only do these bats have to be exquisitely sensitive to the soft, low frequency sounds produced by walking/rustling prey, they also have to precisely localize these sounds. Gleaners typically use low intensity echolocation calls. Such stealth echolocation requires a nervous system that is attuned to low intensity sound processing. In addition, landing on the ground to hunt may bring gleaners in close proximity to ven-
\end{abstract}

\section{KARGER}

() 2018 S. Karger AG, Basel

E-Mail karger@karger.com

www.karger.com/bbe omous prey. In fact, at least 2 gleaning bat species are known to hunt highly venomous scorpions. While a number of studies have addressed adaptations for echolocation in bats that hunt in the air, very little is known about the morphological, behavioral, and neural specializations for gleaning in bats. This review highlights the novel insights gleaning bats provide into bat evolution, particularly auditory pathway organization and ion channel structure/function relationships. Gleaning bats are found in multiple families, suggesting convergent evolution of specializations for gleaning as a foraging strategy. However, most of this review is based on recent work on a single species - the pallid bat (Antrozous pallidus) - symptomatic of the fact that more comparative work is needed to identify the mechanisms that facilitate gleaning behavior.

(c) 2018 S. Karger AG, Basel

\section{Introduction}

The vast majority of bat species use echolocation for general orientation and foraging while in flight [Griffin, 1958; Schnitzler et al., 2003]. This behavior is termed "aerial hawking." A comparison of the properties of emitted pulses and returning echoes is used to determine various features of prey, including distance, velocity, and size to hunt in flight [Simmons et al., 1975; Suga, 1984; Moss 


\begin{tabular}{ll}
\hline Abbreviations used in this paper \\
\hline CF & characteristic frequency \\
CL & contralateral \\
DRG & dorsal root ganglion \\
FM & frequency modulated \\
FMSR & FM sweep selective region \\
IC & inferior colliculus \\
ICc & central IC \\
IID & interaural intensity differences \\
IL & ipsilateral \\
MGB & medial geniculate body \\
MGBd & dorsal MGB \\
MGBv & ventral MGB \\
NSR & noise selective region \\
SG & suprageniculate nucleus \\
\hline
\end{tabular}

and Sinha, 2003]. The utility of various types of echolocation calls in specific ecological niches [Neuweiler, 2003], and the neural adaptations for processing echoes are subjects of excellent reviews [Suga, 1989; Neuweiler, 1990; Kössl et al., 2015]. These studies have provided insights into the convergence of niche-specific signal design and also point to the flexibility of echolocation call properties to aid context-dependent behaviors [Moss and Surlykke, 2010].

From the perspective of prey, studies have examined predation avoidance behaviors such as flight maneuvers, startle, and the production of ultrasonic signals either for jamming or for mimicry [Roeder, 1967; Barber and Conner, 2007; Corcoran et al., 2009; Kawahara and Barber, 2015; ter Hofstede et al., 2015]. Eared prey detect echolocation calls, leading to defensive behaviors. The distance at which the bat is detected depends, in part, on the intensity and rate of echolocation calls. Aerial hawking bats typically produce relatively loud calls ( $>100 \mathrm{~dB}$ SPL) to obtain sufficiently loud echoes from small prey [Surlykke and Kalko, 2008]. The bats also increase the rate of calling as they approach prey in a behavior aptly named the "terminal buzz." While these characteristics increase the probability of detection and hunting of small prey, they also make the bat conspicuous to eared prey at distances sufficient to mount appropriate defenses [Roeder, 1962, 1967].

A subset of bat species utilizes a foraging strategy called "substrate gleaning." These bats forage for surface-dwelling (ground, foliage, etc.) prey and utilize a combination of echolocation, vision, and prey-generated sounds to detect, localize, and hunt prey [Griffin, 1958; Bell, 1982; Bell and Fenton, 1986; Hackett et al., 2014]. Bats that depend primarily on prey-generated noise to hunt have been termed "passive gleaners." The term "passive" is simply used to distinguish this strategy from echolocation. Echolocation is considered "active" sensing because bats perform sensorimotor adjustments to modify emitted pulses to optimize returning echoes. Passive gleaning bats typically produce low intensity echolocation calls and may cease echolocating altogether as they approach prey. Such stealth echolocation may reduce the probability of detection by eared prey and also aid in hunting in clutter or on surfaces that can produce strong interfering echoes. Thus, passive listening for prey-generated sounds is likely a counterstrategy in gleaning bats, which enables a broadening of diet choice to include surface-dwelling eared prey that hides in clutter [Griffin 1958; Faure et al., 1990; Arlettaz et al., 2001; Barber et al., 2003; Russo et al., 2007; Corcoran and Conner, 2017].

Aerial hawking and gleaning are not mutually exclusive foraging strategies as bats can switch depending on prey availability [Hackett et al., 2014] and background clutter [Arlettaz et al., 2001]. Bats can also use low intensity stealth echolocation to hunt prey in the air [Goerlitz et al., 2010; Corcoran and Conner, 2017]. Thus, it is difficult to classify bats as aerial hawkers versus substrate gleaners. Nevertheless, substrate gleaning, primarily by listening to prey-generated noise, necessitates a number of morphological, neural, and behavioral specializations. These adaptations appear in multiple genera of bats indicating convergent evolution related to this foraging strategy. Relative to aerial hawkers, some of the key issues that gleaning bats face include:

1. Segregating echoes and prey-generated noise as distinct acoustic streams that need to be simultaneously localized;

2. Neural and behavioral adaptations for low intensity echolocation that facilitates stealth hunting;

3. Neural and behavioral adaptations for accurate passive sound localization;

4. Overcoming venom of ground-dwelling prey species which can be a major part of gleaning bat diet.

5. This review summarizes recent progress in understanding specializations in gleaning bats that hunt mostly by localizing prey-generated sounds. In particular, this review highlights the neuroethology of passive sound localization, stealth echolocation, and venom resistance primarily based on work in the pallid bat [Bell, 1982; Fuzessery et al., 1993], highlighting the fact that very little comparative work has been done on neural mechanisms of gleaning. 


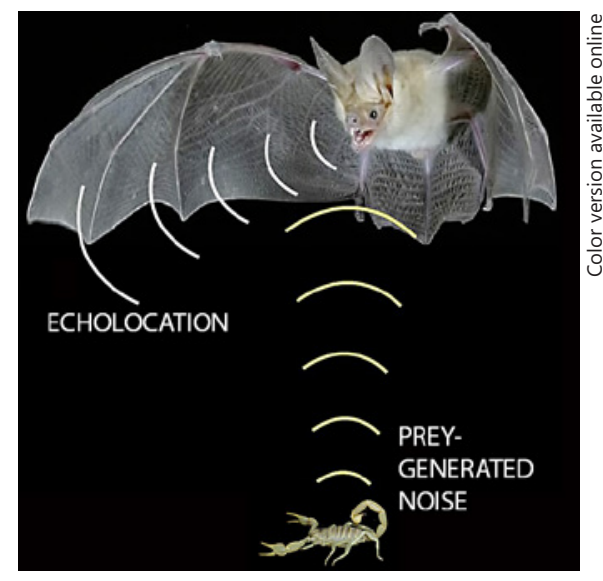

Fig. 1. The pallid bat uses echolocation to detect and avoid obstacles while listening for prey-generated noise to localize and hunt terrestrial prey such as crickets and scorpions. The bat's echolocation call is a downward FM sweep $(60 \rightarrow 30 \mathrm{kHz}$, approx. $3 \mathrm{~ms})$. Prey-generated rustling and walking sounds have energy in the $5-30 \mathrm{kHz}$ band. The pallid bat, as a gleaner, is faced with several unique pressures compared to aerial hawking bats. These include segregated processing of the 2 simultaneous streams of acoustic input (echoes and prey-generated noise), detection of soft echoes and prey-generated noise, precise $2 \mathrm{D}$ localization of prey using passive hearing, and venom as defense in several ground-dwelling prey.

\section{Large Pinna Is a Defining Morphological Feature of Gleaning Bats}

Gleaning bats listen to prey rustling, walking, and calling sounds to localize and hunt. Insect walking and rustling sounds typically have energy in the $5-30 \mathrm{kHz}$ range [Goerlitz et al., 2008]. Gleaning bat audiograms show extreme sensitivity to frequencies between 10 and $20 \mathrm{kHz}$ [Neuweiler et al., 1984; Guppy and Coles, 1988; Coles et al., 1989]. An obvious morphological feature that distinguishes gleaning bats is their large pinnae. Studies of ear directionality suggest that the pinna of gleaning bats [Guppy and Coles, 1988; Coles et al., 1989; Obrist et al., 1993; Fuzessery, 1996] provide considerable gain (approx. $20 \mathrm{~dB}$ SPL) for frequencies of approximately 10-20 $\mathrm{kHz}$. The size of the ear is similar to the wavelength of sounds at these frequencies and thereby the pinna provides resonances that increase gain. Removal of pinna reduces the gain. At these frequencies, the ear is also broadly directional. The ear directionality sharpens with increasing frequency, with very sharp directionality at the higher frequencies used in passive hearing (approx. 20$30 \mathrm{kHz}$ ). Therefore, the bats obtain large interaural intensity differences (IID) at these higher frequencies in prey-

Adaptations for Substrate Gleaning in

Bats generated noise. This suggests that the pinna morphology is adapted to aid both detection (high gain and broad directionality) and precise localization (large IIDs, see below) using frequencies in the $5-30 \mathrm{kHz}$ prey-generated noise. Consistent with this prediction is that passive horizontal sound-localization accuracy of the pallid bat declines if frequencies $>20 \mathrm{kHz}$ are removed from target noise [Brewton and Razak, 2018]. One downside of large ears is reduced aerodynamic performance [Fenton, 1972; Gardiner et al., 2011; Håkansson et al., 2017]. Large-eared bats have higher body drag and gleaners, in general, may not be able to perform high-speed aerial acrobatics. However, the large ears may contribute to lift generation in slow flight [Håkansson et al., 2017], which gleaners show [Norberg and Rayner, 1987]. Thus, ear morphology contributes not just to acoustic amplification of relevant frequencies, but also to flight behaviors involved in surface gleaning.

\section{Auditory System of Gleaning Bats: The Pallid Bat as a Case Study}

The auditory systems of gleaning bats that depend primarily on prey-generated sounds to hunt have received much less attention compared to aerial hawking bats. Thus far, only the false Indian vampire bat (Megaderma lyra), pallid bat (Antrozous pallidus) and Waterhouse's leaf-nosed bat (Macrotus waterhousii) has been studied in terms of brain organization. Much of this work has been conducted on the pallid bat. The pallid bat depends primarily on localizing prey-generated noise to hunt (Fig. 1). Its echolocation call is a short duration $(2-4 \mathrm{~ms}), 60 \rightarrow 30$ $\mathrm{kHz}$ downward frequency modulated (FM) sweep. Echolocation is primarily used for obstacle avoidance and general orientation. The pallid bat auditory system is described here to highlight the specializations as potentially general adaptations in gleaners. The pallid bat can then be compared to aerial hawking bats, many of which are well described in the literature (e.g., mustached bat, Mexican free tailed bat, little brown bat, and big brown bat). Such a comparison will highlight differences in neural mechanisms likely related to different foraging strategies [Neuweiler, 1990].

\section{Parallel Pathways to Enhance Dual Processing}

The major conclusion of studies that examined the auditory system of the pallid bat is that there are parallel pathways dedicated to processing echolocation calls and the prey-generated noise (Fig. 2). 


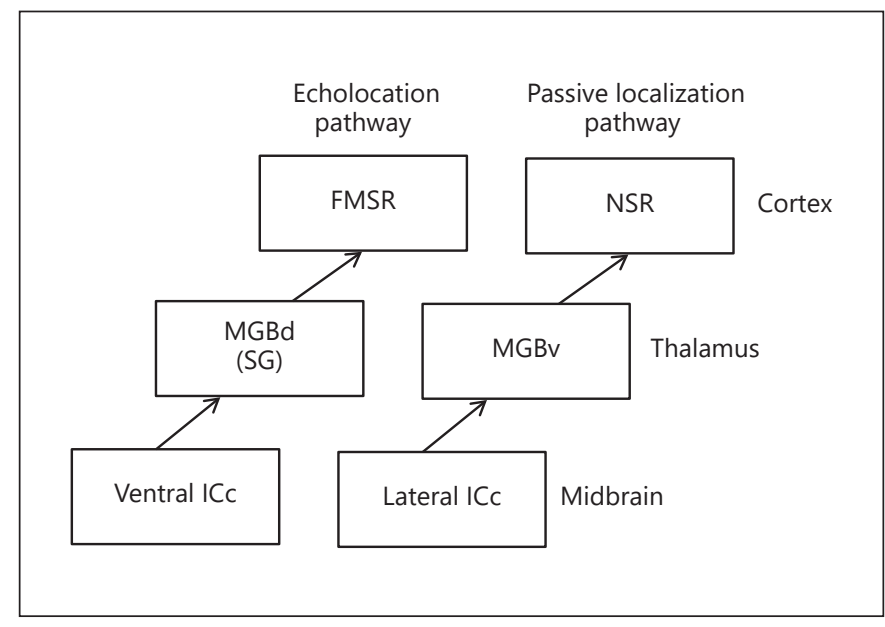

Fig. 2. Parallel pathways are used to process the 2 different sounds used in echolocation and passive gleaning. The IC and cortical neurons in the echolocation pathway respond selectively, and sometimes exclusively, to the downward FM sweep used in echolocation. This pathway is routed through the dorsal division of the MGB (mainly the SG). The IC and cortical neurons in the passive gleaning pathway are selective for prey-generated noise and are routed through the ventral MGB.

Inferior Colliculus

The inferior colliculus (IC) of the pallid bat consists of 3 functionally distinct regions [Fuzessery, 1997; Fuzessery and Hall, 1999]. The ventral part of the central IC (ICc), tuned to frequencies $>30 \mathrm{kHz}$, is specialized to process the downward FM sweeps used in echolocation. Most neurons in this region respond best, or exclusively, to bandwidths, rates, and direction of FM sweeps present in the echolocation call. Neurons in the lateral ICc are tuned $<30 \mathrm{kHz}$ with many neurons showing preferential responses to low-frequency noise. This suggests that lateral ICc may be involved in prey-localization behavior. Neurons in the dorsal IC are also tuned $<30 \mathrm{kHz}$, but the functional significance of this region is unclear because only a few neurons show response to noise or echolocation calls.

Data from the false Indian vampire bat (M. lyra) further support the suggestion that the lateral IC is specialized for processing prey-generated noise in gleaning bats. Rübsamen et al. [1988] found an overrepresentation of frequencies $<20 \mathrm{kHz}$ in the lateral IC of M. lyra. Neurons in this region show low thresholds $(<30 \mathrm{~dB}$ SPL) and strong or even exclusive response to noise. Many of these neurons also show narrow tuning curves and nonmonotonic rate intensity functions, indicating specificity for low intensity, low frequency sounds. These features of IC responses were interpreted as adaptations for detecting prey-generated noise.

The IC organization in gleaners is in contrast to bats that use characteristic frequency (CF)-FM type echolocation calls to hunt, such as the mustached bat (Pteronotus parnellii), which overrepresents the approximately 60 $\mathrm{kHz}$ emphasized in the echolocation call. The IC of $M$. lyra and A. pallidus is also distinct from bats that use FM type echolocation calls to hunt, such as the Mexican freetailed bat (Tadarida brasiliensis) [Bodenhamer and Pollak, 1981] and big brown bat (Eptesicus fuscus) [Jen and Schlegel, 1982], which have a more uniform representation of the audible range. These comparisons suggest FM/ nongleaners are similar to nonchiropteran mammals with a uniform tonotopy. The CF-FM bats disrupt uniform tonotopy by emphasizing the $\mathrm{CF}$ component while the FM/gleaners emphasize, particularly in the lateral ICc, frequencies present in prey-generated noise.

\section{Thalamus}

The physiological properties of the medial geniculate body (MGB) have not been studied in any gleaning bat and this remains a critical gap in our understanding of the evolution of gleaning in bats. However, thalamocortical connections have been studied in the pallid bat (see below), further supporting the idea of parallel pathways for echolocation and prey localization.

\section{Auditory Cortex and Thalamocortical Connections}

The auditory cortex of the pallid bat contains a tonotopic map with the audible range (approx. 5-70 kHz). The frequency representation has 2 peaks centered at approximately 15 and $40 \mathrm{kHz}$, corresponding to the sounds used in passive localization and echolocation, respectively. Overlain on this map are 2 mostly segregated regions with selectivity for sounds used in the 2 behaviors [Razak and Fuzessery, 2002]. The FM sweep selective region (FMSR), like the ventral ICc, contains neurons with tuning $>25 \mathrm{kHz}$ and responds selectively to the bandwidths, rates, and direction of FM sweeps in the echolocation call [Razak and Fuzessery, 2006; 2008; Razak et al., 2008]. The noise selective region (NSR), like the lateral ICc, contains neurons tuned $<35 \mathrm{kHz}$ and responds selectively to noise with energy between 5 and $35 \mathrm{kHz}$ [Razak 2011, Razak et al., 2015]. Thus, the segregated functional organization observed in the IC is continued in the auditory cortex.

Perhaps the most convincing evidence for parallel pathways for echolocation and passive hearing is from studies that traced connections from the thalamic MGB to the auditory cortex. By using dual tracer injections in 
physiologically identified cortical regions, Razak et al. $[2007,2009]$ showed that the FMSR receives inputs from the dorsal MGB (MGBd), particularly from the suprageniculate nucleus (SG). The NSR, on the other hand, receives tonotopically organized inputs from the ventral MGB (MGBv). The FMSR, which represents one half of the audible range of pallid bats, does not receive input from the MGBv. This is a significant deviation from the general mammalian plan, including aerial hawking bats, wherein the MGBv represents almost all of the audible range of a species and projects it to the primary auditory cortex. In the pallid bat, it appears that the low and high frequency halves of the audible range are routed through 2 distinct divisions of the MGB, suggesting that segregated processing of echolocation calls and prey-generated noise is prioritized in this auditory system. Electrophysiological recordings from the MGB are required to confirm this. Preliminary data also suggest that the lateral and ventral ICc also connect to the MGBv and MGBd, respectively [Razak and Fuzessery, unpubl. observations]. Together, these data show the existence of parallel pathways from the IC-MGB-cortex with physiological properties specialized to extract information about echoes and prey-generated noise (Fig. 2). Thus, in contrast to aerial hawking bats that show segregated cortical regions to extract prey-specific information such as distance and velocity [reviewed in Suga, 1990], the pallid bat shows segregated cortical regions for 2 different behaviors - active echolocation and passive prey localization.

Whether such parallel pathways are seen in other gleaning bats is not known. As mentioned earlier, the IC has been examined only in M. lyra. The auditory cortex has been studied in only 1 other species of gleaning bat. $M$. waterhousii, an insectivorous bat in the family Phyllostomidae [Macías et al., 2014]. There is a continuous tonotopic map in the cortex with no overrepresentation of any specific frequency bands. Because response selectivity was not studied using broadband sounds, it is not clear if functionally segregated regions are present. Thus, future studies are needed to compare neural pathways used in echolocation and passive hearing in other gleaners to understand the evolution of this foraging strategy in bats.

\section{Functional Significance of Parallel Pathways in Gleaning Bats}

The pallid bat data support the idea that parallel pathways are used for echolocation and passive hearing. While different frequency ranges are used for these 2 behaviors, the parallel pathways are not simply a reflection of tono-

Adaptations for Substrate Gleaning in

Bats topic representation. Two features of auditory system organization in the pallid bat best exemplify this. First, the echolocation pathway is routed through a distinct division of the MGB (dorsal, mainly through the SG). The MGBv contains the passive hearing pathway. This is an unusual organization because in most mammals studied, including other bats, the MGBv represents the full audible range of the species. In the pallid bat, the MGBv does not project to almost one half of the cortical tonotopic map. Second, the level of FM selectivity in the IC and AC in pallid bats is unusual, even for bats. The majority of IC neurons tuned $>30 \mathrm{kHz}$ respond selectively to the FM sweep used in echolocation, while a third respond almost exclusively to it [Fuzessery, 1994]. This is in contrast to aerial hawking bats, in which $<10 \%$ of IC neurons respond selectively to the echolocation pulse [Suga 1965, 1969; O’Neill 1985]. A similar degree and prevalence of selectivity is also present in the cortex of the pallid bat [Razak and Fuzessery, 2002].

The strong selectivity for FM sweeps and noise acts as biological filters that segregate the neural pathways serving echolocation and prey localization. These parallel pathways in the pallid bat may be a solution to the problem of auditory stream segregation, a process by which animals separate acoustic information from multiple sources that arrive simultaneously. Gleaning bats are faced with the task of processing 2 distinct streams of auditory inputs (prey-generated noise and echoes) that arrive simultaneously. The differences in frequencies (low vs. high), spectrotemporal structure (noise vs. FM) and spatial locations (ground vs. flight path) between the 2 sounds will aid stream segregation [Bregman, 1990]. In addition, parallel auditory pathways will enhance dual stream processing by ensuring adequate neural resources are available to process both sounds. Because bats have control over when they use echolocation, they can also alter call production rates to reduce the potential overlap between streams of echoes and prey-generated noise. The pallid bat does this when forced to process the 2 streams simultaneously [Barber et al., 2003]. This is also seen in other gleaning bats, which reduce echolocation call rates when they glean [Arlettaz et al., 2001]. This is in contrast to aerial hawkers, which increase the calling rate as they get closer to prey (terminal buzz). The reduced use of echolocation while gleaning may thus serve 2 purposes: reduced detection by eared prey and enhanced stream segregation. Thus, the pallid bat appears to use acoustic, neural, and behavioral mechanisms to enhance stream segregation. Whether similar parallel pathways are present in other gleaners remains unclear, although the $M$.

Brain Behav Evol 2018;91:97-108 
lyra IC data suggest that this may be present. A particularly important question is if the MGBd (SG, in particular) is a phylogenetically plastic region that is used by gleaning bats to route the echolocation pathway, while leaving the MGBv for passive hearing. Additional gleaning bats need to be studied to explore these issues. Responses below the level of the midbrain have also not been analyzed in any gleaning bat. Therefore, the level of the auditory system at which responses become specialized for the 2 behaviors remains unclear.

\section{Stealth Hunting and Neural Specializations for "Whispering"}

Gleaning bats use low intensity echolocation calls and have been referred to as "whispering bats" [Griffin, 1958]. Compared to most aerial hawkers whose echolocation call intensities are $>100 \mathrm{~dB}$ SPL, gleaning bat calls are typically $<80$ dB SPL [Waters and Jones, 1985; Holderied et al., 2011; Measor et al., 2017]. Passive gleaning may have indeed evolved to reduce the probability and distance of detection by eared prey by reducing dependence on high intensity echolocation. Low intensity echolocation may also aid hunting when bats glean in cluttered environments [Korine and Pinshow, 2004]. However, it must be noted that some species of gleaners show context-dependent echolocation behaviors and are capable of producing louder calls. Likewise, bats in the aerial hawking mode can also use low intensity echolocation calls to reduce detection by eared prey [Goerlitz et al., 2010; Corcoran and Conner, 2017]. Faure et al. [1993] for example, showed that Myotis evotis call intensities are approximately $77 \mathrm{~dB}$ SPL when gleaning. Eared moth auditory neurons fail to respond above spontaneous rates for these calls. Moths also failed to show behavioral responses to these calls. However, M. evotis also hunt in the air, during which the call levels are approximately $90 \mathrm{~dB}$ SPL and include call features typical of aerial hawkers such as terminal buzzes [Faure and Barclay, 1994]. Likewise, Otonycteris hemprichii produces calls that are approximately $80 \mathrm{~dB}$ SPL while foraging by gleaning. However, these bats can produce calls $>110 \mathrm{~dB}$ SPL when hunting aerial prey indicating plasticity in foraging strategy and call intensities [Hackett et al., 2014]. More recently, Corcoran and Conner [2017] reported that Corynorhinus townsendii uses calls with levels of approximately 80 or 93 $\mathrm{dB}$ SPL, depending on the foraging conditions.

The central auditory system of gleaning bats must be adapted to process very low intensity echoes. A recent study of the pallid bat revealed an echolocation system that is specialized to process sounds with intensities between 30 and $50 \mathrm{~dB}$ SPL [Measor et al., 2017]. The pallid bat uses downward FM sweeps to echolocate. Its echolocation calls are approximately $75-80 \mathrm{~dB}$ SPL. Similar to many aerial hawking bats, the pallid bat performs echo intensity compensation wherein the intensity of emitted calls is reduced as it approaches a target. The echoes that the pallid bat receives are thus maintained at a relatively constant intensity between 40 and $50 \mathrm{~dB}$ SPL. The vast majority of FMSR neurons in the auditory cortex of the pallid bat are intensity tuned. They have a nonmonotonic relationship between sound intensity and response rate, with a distinct peak (best intensity). Responses decline with increasing sound intensities. Remarkably, most neurons have thresholds $<30 \mathrm{~dB}$ SPL and show a rapid increase in response magnitude with intensity (dynamic range approx. $10 \mathrm{~dB}$ ). The best intensity of most FMSR neurons is $<50 \mathrm{~dB}$ SPL. The slope of the rate-intensity curve with increasing sound intensities is also in the 30$50 \mathrm{~dB}$ SPL range. This indicates that the FMSR provides most information in the range of intensities which the bat actively maintains using echo intensity compensation behavior. In M. waterhousii, the only other gleaning bat in which sound intensity representation has been studied, Macías et al. [2014] also reported that most cortical neurons represent a narrow range of sound levels. It was suggested that this relates to the capacity of the bat to maintain echoes in a certain range by active sensorimotor adjustments, consistent with the pallid bat data. However, whether these bats show echo intensity compensation is not clear. Unlike the mustached bat [Suga, 1977], neither of the 2 gleaners showed any systematic representation of best intensities (amplitopy) in the auditory cortex. The pallid bat data show that there are coordinated neural and behavioral adaptations in a gleaning bat for stealth echolocation and low intensity processing. Whether these coordinated neural and behavioral adaptations are present early in development, or if experience with echolocation shapes such matching, remains unclear.

\section{Neuroethology of Sound Localization}

Gleaning bats face a sound-localization problem analogous to that faced by barn owls - precise localization of faint rustling/walking sounds of prey in the dark. Therefore, it can be predicted that gleaning bats should have accurate passive sound-localization behavior, but only the pallid bat has been studied for this purpose. In the pallid bat, passive localization accuracy is between 3 and $5^{\circ}$ 


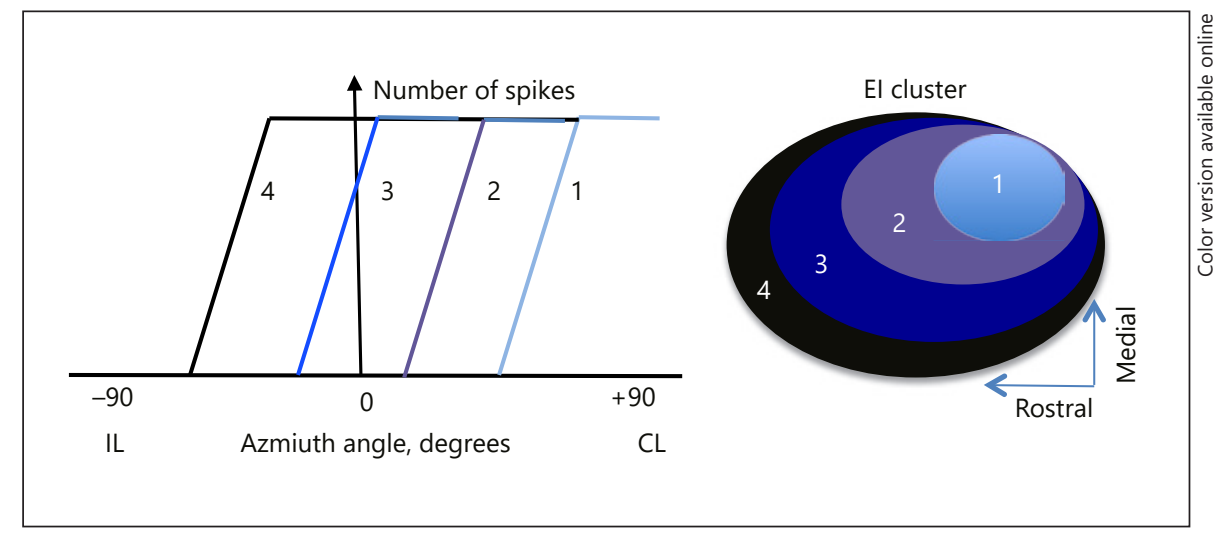

Fig. 3. The extent of EI cluster activation changes systematically with azimuth location in the NSR of the pallid bat auditory cortex. Left: azimuth selectivity of 4 example neurons. The azimuth angle at which response declines to a minimum (azimuth cut-off) is different for these 4 neurons. The azimuth cut-offs for example neurons $1-4$ are approximately $-60^{\circ},-20^{\circ},+15^{\circ}$, and $+45^{\circ}$, respectively. Right: a schematic of the arrangement of the azimuth cutoffs in the EI cluster of the NSR of the pallid bat auditory cortex. The azimuth cut-off angle of neurons varies systematically in the EI cluster of the NSR such that neurons with a highly CL cut-off

for broadband noise [Fuzessery et al., 1993; Barber et al., 2003; Brewton and Razak, 2018]. This is comparable to the accuracy reported in the barn owl [Knudsen et al., 1979] and only a few mammals are more accurate (e.g., humans have approx. $2^{\circ}$ localization accuracy). Thus, the pallid bat is a suitable species to study specializations for sound localization that may facilitate an understanding of general principles in mammalian spatial encoding. In the only other gleaning bat studied, M. lyra, lateralization, but not localization, was measured. This species can lateralize sound sources around midline with a $2^{\circ}$ accuracy for $20 \mathrm{kHz}$ pure tones, and between 4 and $7^{\circ}$ accuracy for lower frequencies [Witzke, 1987]. This suggests that passive sound localization will be accurate, particularly for broadband noise, but this needs to be examined.

Recent studies of the pallid bat cortex provide some insights into the mechanisms underlying such accurate localization. These studies show that the $2 \mathrm{D}$ location representation in the auditory cortex is fundamentally different than in the midbrain [Razak and Fuzessery, 2002; Razak 2011, 2012, 2016; Razak et al., 2015]. Pallid bats fly with their ears facing downwards (pinna parallel to ground) when foraging. Elevation of a source on the ground is in a direction parallel to the long axis of the bat's body and azimuth of the source perpendicular to the bat's long axis (2D location on the ground). We have studied (e.g., light blue line on the left, "1") forms a small cluster which is surrounded in a semiconcentric fashion by neurons with increasingly more IL located cut-offs (e.g., neurons 2, 3, and 4 on the left). Thus, when a sound arrives from $-45^{\circ}$, only the neurons shown in black will be activated. As the sound moves to $+45^{\circ}$, all but the light blue shaded regions (neuron 1) will be activated. When the sound is in an extreme CL location, the entire EI cluster will be activated. Thus, the extent of active cortex will change in a predictable manner as the sound source azimuth changes from IL to CL locations. how $2 \mathrm{D}$ locations of prey-generated noise are represented in the auditory cortex. One reason to focus on the auditory cortex is that the cortical mechanisms of sound localization are not known. The one-to-one topographical space map described in the barn owl [Knudsen, 1982] and mammalian [King and Hutchings, 1982] midbrain is not seen in any auditory cortex [Stecker and Middlebrooks, 2003; King et al., 2007]. While this suggests a different mechanism in the cortex, the nature of such representations is not known.

The focus of analyses in the pallid bat has been on the NSR with neurons tuned between 5 and $35 \mathrm{kHz}$ because this region likely underlies passive sound localization. We recorded sensitivity to IID because of ear directionality studies that showed strong frequency and location-specific IIDs up to $\pm 25 \mathrm{~dB}$ generated by the pallid bat. Studies of IID sensitivity in NSR neurons showed 2 main types of responses: peaked and EI. Peaked neurons are those with strong responses to binaural stimuli with IID of approximately $0 \mathrm{~dB}$. These neurons respond poorly to sounds that favor one or the other ear in intensity. EI neurons show sigmoidal IID selectivity and are excited by stimuli that favor the contralateral (CL) ear in intensity (examples are provided in Fig. 3). As the level in the ipsilateral (IL) ear increases, the response is inhibited (giving rise to the name "EI" neurons). Different neurons are inhibited 


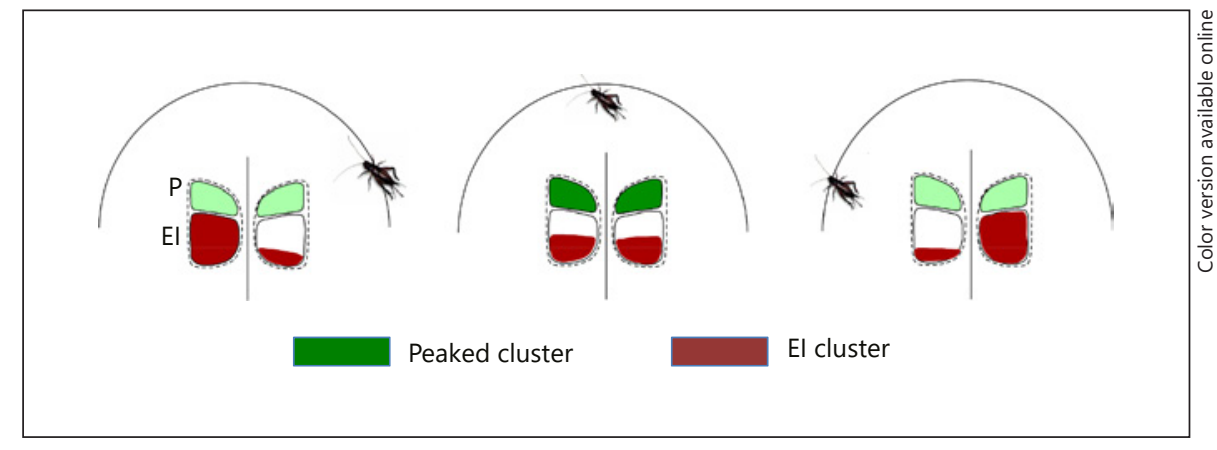

Fig. 4. Simplified schematic of proposed azimuth localization code for azimuth localization of prey-generated noise in the pallid bat. The 3 panels show predicted activity of peaked and EI clusters on both hemispheres as the source (prey) moves from eccentric (left and right panels) to midline (middle panel) locations. The dashed contour is the NSR in the auditory cortex. The vertical line separates the 2 cortical hemispheres. The peaked clusters are fully activated only when the prey is near the midline. The EI cluster is

at different IIDs; this value is known as the inhibitory threshold. The peaked and EI neurons are represented in 2 separate clusters within the NSR.

Azimuth selectivity of peaked and EI neurons is predicted by IID responses and frequency tuning [Razak, 2011, 2012]. Almost all peaked neurons respond best to frontal azimuth space between 0 and $15^{\circ}$, indicating the presence of a separate cluster of neurons within the NSR specifically to represent the midline azimuth. This predicts that the pallid bat should show high acuity sound localization near the midline. Similar midline preferring neurons are also seen in other mammals [Stecker et al., 2005; Higgins et al., 2010] and may explain the generally better localization accuracy near midline compared to more eccentric locations [Nodal et al., 2008]. In EI neurons, response is strong for CL locations and weakens as the sound moves to more IL locations. The inhibitory threshold of IID predicts the azimuth at which the neuron's response declines to a minimum (azimuth cut-off). Interestingly, the azimuth cut-off of EI neurons is arranged in a systematic manner. The arrangement is such that neurons with cut-offs in high eccentricity CL locations are surrounded in a semiconcentric form by neurons with progressively more IL cut-offs (Fig. 3). Therefore, when prey-generated noise arises from IL locations, only a few neurons are excited. However, as the sound moves to more and more CL locations, a systematically increasing extent of sigmoidal cluster neurons is included in the activity map. Thus, the extent of active neurons represents the azimuth angle within the NSR. only partially activated when prey is at the midline because azimuth cut-offs of nearly half the EI neurons are located in the CL hemifield, i.e., nearly half of the EI neurons are inhibited when sound is at the midline when the peaked neurons are fully activated. For any eccentric location of prey, the difference in activation of the 2 EI clusters indicates the azimuth angle and the sensorimotor transformation needed.

Together, the peaked and EI cluster in the 2 hemispheres can represent the full range of azimuth angles. When the prey is at a peripheral azimuth location, the CL EI cluster is fully active while the IL EI cluster is only weakly active (Fig. 4, left and right). Both peaked clusters are weakly active. The degree to which the bat has to turn (sensorimotor transformation) to face the prey is related to the difference in extent of activation of the 2 EI clusters. Once the bat faces the prey, the peaked clusters on both sides are fully active and the EI clusters are equally active, albeit not fully (Fig. 4, middle). The bat will then be on target as long as the 2 peaked clusters are fully activated. This suggests a mechanism of azimuth localization that depends on comparing the activity between the binaural clusters on both cortical hemispheres. This remains to be tested behaviorally.

We also examined how elevation of the source affects spatial tuning within the NSR. Only the EI cluster was examined in this study [Razak et al., 2015]. By recording responses to broadband noise presented from 55 speakers, 2 major trends emerged. First, neurons with low CF had broad elevation tuning and neurons with high CF exhibited sharper elevation tuning. Second, the centroid of elevation tuning shifted to higher elevations with increasing CF. Together, these data indicate an elevation representation that depends on the tonotopic arrangement within the NSR. For broadband noise that originates from low elevations, peak activity will be restricted to low CF neurons in the NSR. As source elevation rises, the low CF neurons will continue to be active (because of 


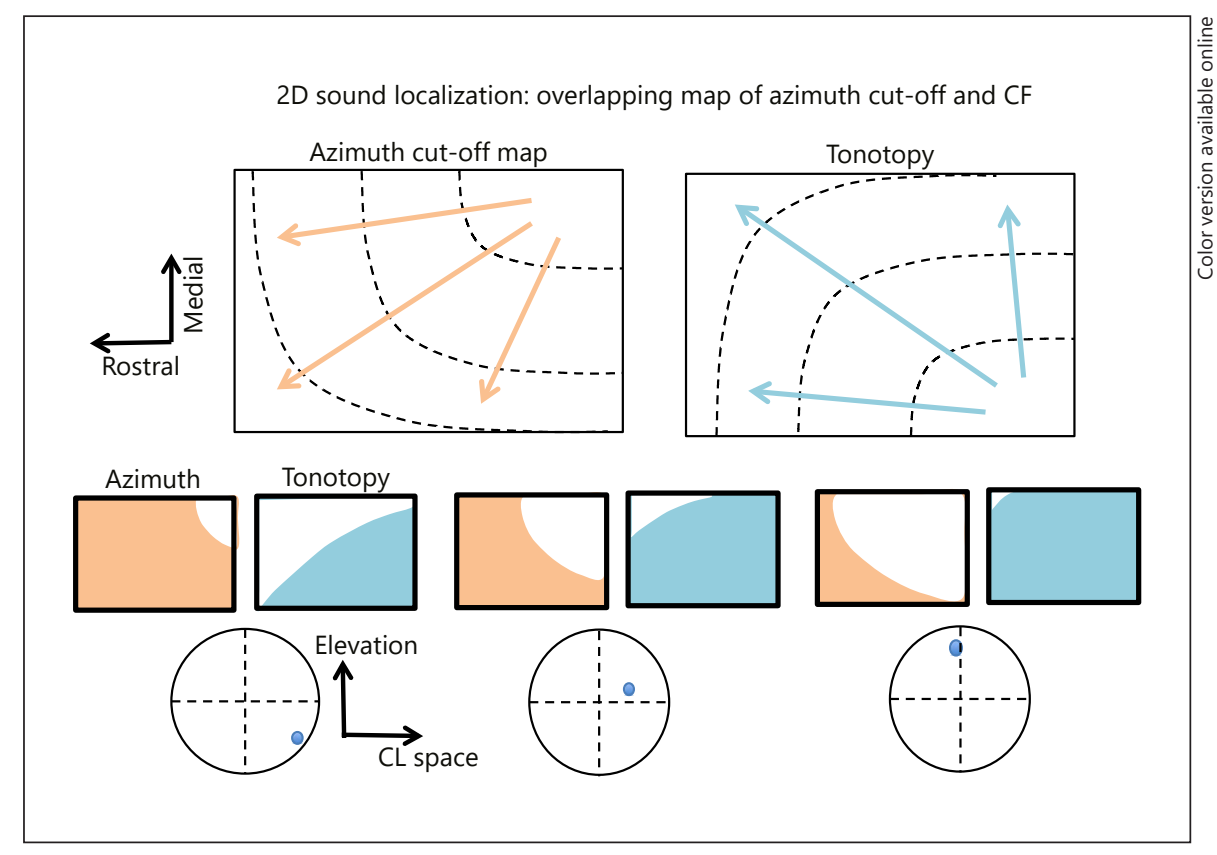

Fig. 5. The 2D location of prey is represented in the auditory cortex by activation patterns dictated by overlapping azimuth cut-off and tonotopic representations. The large rectangles represent the CL EI cluster of the NSR in the pallid bat auditory cortex. The rectangle on the left indicates the azimuth cut-off map, while the map on the right is a representation of frequency tuning. The smaller rectangles illustrate activity patterns in the same maps (azimuth cut-off and tonotopy) for 3 different prey locations. The 3 different prey locations are illustrated by the blue dot in the circles that represent the $2 \mathrm{D}$ sound field on the ground, with the bat looking down such that its nose is facing the middle of the circle. The blue dot represents prey moving from a high CL angle, low elevation location towards the midline azimuth, and high elevation location at 3 different time points. The middle row of panels (small rectangles) shows the extent of activity in the EI cluster for the different azimuth and elevation locations of the prey. As the azimuth location moves to more CL space, an increasing area of the EI cluster will be activated. As the elevation angle increases from below to above the nose, more of the EI cluster will be activated based on tonotopy. Any given prey location will therefore activate a unique combination of overlapping azimuth and tonotopic maps. See the main text for more details. broad elevation tuning), but more of the high CF neurons will be recruited.

When considered together, the map of azimuth cut-off and CF are arranged more or less orthogonal to each other (Fig. 5). The azimuth source location will dictate how much of the NSR will be active based on azimuth cut-off representation. The elevation of the source will dictate how much of the NSR will be active based on frequency representation (tonotopy). Thus, for any 2D source location, the extent and loci of activity in the NSR will be dictated by the nature of overlap between tonotopy and azimuth cut-off maps. This is the first proposal for how 2D sound locations are represented in a mammalian auditory cortex. This is different from how the midbrain represents locations. In the midbrain, the one-to-one topographic space map will restrict the focus of active neurons to a small region depending on sound location. Only a small percentage of midbrain neurons will be active. In

Adaptations for Substrate Gleaning in

Bats the cortex, the extent of active neurons represents location, but most of the cortex will be active for any CL location. This may be related to the distinct functions of the midbrain and cortex, with the former involved in reflexive orientation to sound location. The latter is not just involved in sound localization, but also in identifying other characteristics of the source. The way the pallid bat solves its localization problem may guide a better understanding of how and why the auditory cortex represents sound locations in a fundamentally different manner to the midbrain.

\section{Resistance to Scorpion Venom}

A significant selective pressure against gleaning as a foraging strategy is the probability that the rustling noise on the ground is produced by venomous critters. In the 
darkness, unless the bat can use passive hearing or echolocation to identify its prey, it may land on venomous prey. At least 2 gleaning bats are known to hunt highly venomous scorpions. $O$. hemprichii has been documented to hunt the death stalker scorpion [Holderied et al., 2011]. The pallid bat hunts the Arizona bark scorpion, North America's most venomous scorpion [Hopp et al., 2017]. It may be possible that the bats exhibit behavioral techniques to avoid the stinger, but a recent study of the pallid bat showed that the bat is stung multiple times by the scorpion during the hunt [Hopp et al., 2017]. Likewise, $O$. hemprichii also did not show any risk-averse behaviors when dealing with scorpions of different venom toxicity. These bats land directly on the scorpions and appear to be resistant to the venom. This was confirmed by injecting Arizona bark scorpion venom directly into the pallid bat with no effect on behavior and survival. A dose of approximately $10 \times$ the LD50 (lethal dose 50 , the dose that kills $50 \%$ of animals tested) for a house mouse produces no effect on the pallid bat.

Scorpion venom is a mixture of peptides, some of which bind to voltage-gated sodium ion channels (Nav) in sensory neurons of the dorsal root ganglion (DRG). Hyperactivation of these sensory neurons leads to increased pain perception. It appears that the gleaning bats have adaptations in the DRG sensory neurons that reduce pain caused by venom, while not influencing normal pain perception necessary to avoid tissue damage. A recent study of the grasshopper mouse, a rodent that hunts the Arizona bark scorpion, showed a specific mutation in the Nav1.8 channel that essentially reduces sensory neuron firing in the presence of venom. An analysis of the transcriptome of the pallid bat DRG indicates the mechanism seen in the grasshopper mouse is not present. Thus, the pallid bat has solved the venom problem using a hitherto unknown mechanism. A number of mutations in in the pallid bat DRG Nav1.7 that may prevent binding of venom toxins have been identified [Hopp et al., 2017], but the mechanisms of action remain unclear. Interestingly, some of these mutations are present in all bats examined, compared to other mammals, suggesting that Nav1.7 changes common in bats may provide some resistance to venom across bats, but evolved for a different reason and thus expand their diet to include ground-dwelling prey. Gleaning bats may have built upon this and evolved additional mechanisms to enable scorpion hunting. Alternately, other bats that are not known to hunt scorpions may be resistant to venom. Future studies are needed to test to what extent scorpion venom resistance is prevalent in bats.

\section{Outstanding Questions for Future Research}

Clearly, additional passive gleaning bats have to be studied to determine whether gleaners across families have evolved similar or different solutions as the pallid bat for the issues gleaners face compared to aerial hawking bats. Such studies in bats will provide a unique opportunity to address the extent to which auditory system organization can be modified to facilitate different foraging strategies. In particular, the organization of the dorsal MGB and the suprageniculate nucleus provides a good starting point because of the known connectional differences in this region in terms of gleaners and aerial hawking bats. In addition, sound-localization behaviors in gleaners have to be studied to determine if the mechanisms seen in the pallid bat are present in other species. Another outstanding question is how social call processing is organized within the dual pathway scheme. The pallid bat has a rich repertoire of social calls. Is there a separate pathway for social call processing, or is social call processing distributed within the 2 pathways? There have been no studies yet of social call processing at any level of the auditory system in a gleaning bat. Future studies should address this important issue.

In terms of venom resistance, the mechanisms need to be elucidated. While the focus on Nav1.7/Nav1.8 in the DRG addresses only the pain aspect of venom, other aspects of physiology, such as muscle function and sympathetic responses, may also be affected by venom. If and how gleaners evolved multiple lines of defense against venom remains unclear. Whether similar or different mechanisms of venom resistance are present across gleaners remains unclear. If changes in ion channels evolved for a different reason in bats, but was exapted for hunting scorpions remains unclear. These questions call for a concerted effort to perform comparative DRG transcriptome or genome analyses across a number of bat species with different foraging strategies.

\section{Acknowledgements}

Much of the work on the pallid bat reviewed here was done with funding provided by the NIH and NSF. I thank Dr. Zoltan Fuzessery and Dr. Jesse Barber for many discussions that led to the ideas reviewed here.

\section{Disclosure Statement}

The author has no conflicts of interest to declare. 


\section{References}

Arlettaz R, Jones G, Racey PA (2001): Effect of acoustic clutter on prey detection by bats. $\mathrm{Na}$ ture 414:742-745.

Barber JR, Conner WE (2007): Acoustic mimicry in a predator-prey interaction. Proc Natl Acad Sci USA 104:9331-9334.

Barber JR, Razak KA, Fuzessery ZM (2003): Can two streams of auditory information be processed simultaneously? Evidence from the gleaning bat Antrozous pallidus. J Comp Physiol A 189:843-855.

Bell GP (1982): Behavioral and ecological aspects of gleaning by a desert insectivorous bat $\mathrm{An}$ trozous pallidus (Chiroptera: Vespertilionidae). Behav Ecol Sociobiol 10:217-223.

Bell GP, Fenton MB (1986): Visual acuity, sensitivity and binocularity in a gleaning insectivorous bat, Macrotus californicus (Chiroptera: Phyllostomidae). Anim Behav 34:409-414.

Bodenhamer RD, Pollak GD (1981): Time and frequency domain processing in the inferior colliculus of echolocating bats. Hear Res 5: 317-335.

Bregman AS (1990): Auditory Scene Analysis. Cambridge, MIT Press.

Brewton D, Razak KA (2018): Horizontal sound localization performance by a gleaning bat, Antrozous pallidus. Abstract PS 309, Association for Research in Otolaryngology, San Diego, 41st Annual Midwinter Meeting.

Coles RB, Guppy A, Anderson ME, Schlegel P (1989): Frequency sensitivity and directional hearing in the gleaning bat, Plecotus auritus (Linnaeus 1758). J Comp Physiol A 165:269_ 280

Corcoran AJ, Barber JR, Conner WE (2009): Tiger moth jams bat sonar. Science 325:325327.

Corcoran AJ, Conner WE (2017): Predator counteradaptations: stealth echolocation overcomes insect sonar-jamming and evasive-manoeuvring defences. Anim Behav 132:291301.

Faure PA, Barclay RMR (1994): Substrate-gleaning versus aerial-hawking: plasticity in the foraging and echolocation behaviour of the long-eared bat, Myotis evotis. J Comp Physiol A 174:651-660.

Faure PA, Fullard JH, Barclay RM (1990): The response of tympanate moths to the echolocation calls of a substrate gleaning bat, Myotis evotis. J Comp Physiol A 166:843-849.

Faure PA, Fullard JH, Dawson JW (1993): The gleaning attacks of the northern long-eared bat, Myotis septentrionalis, are relatively inaudible to moths. J Exp Biol 178:173-189.

Fenton MB (1972): The structure of aerial-feeding bat faunas as indicated by ears and wing elements. Can J Zool 50:287-296.

Fuzessery ZM (1994): Response selectivity for multiple dimensions of frequency sweeps in the pallid bat inferior colliculus. J Neurophysiol 72:1061-1079.
Fuzessery ZM (1996): Monaural and binaural spectral cues created by the external ears of the pallid bat. Hear Res 95:1-17.

Fuzessery ZM (1997): Acute sensitivity to interaural time differences in the inferior colliculus of a bat that relies on passive sound localization. Hear Res 109:46-62.

Fuzessery ZM, Buttenhoff P, Andrews B, Kennedy JM (1993): Passive sound localization of prey by the pallid bat (Antrozous p. pallidus). J Comp Physiol A 171:767-777.

Fuzessery ZM, Hall JC (1999): Sound duration selectivity in the pallid bat inferior colliculus. Hear Res 137:137-154.

Gardiner JD, Codd JR, Nudds RL (2011): An association between ear and tail morphologies of bats and their foraging style. Can J Zool 89: 90-99.

Goerlitz HR, Greif S, Siemers BM (2008): Cues for acoustic detection of prey: insect rustling sounds and the influence of walking substrate. J Exp Biol 211:2799-2806.

Goerlitz HR, ter Hofstede HM, Zeale MR, Jones G, Holderied MW (2010): An aerial-hawking bat uses stealth echolocation to counter moth hearing. Curr Biol 20:1568-1572.

Griffin DR (1958): Listening in the Dark: The Acoustic Orientation of Bats and Men. New Haven, Yale University Press.

Guppy A, Coles RB (1988): Acoustical and neural aspects of hearing in the Australian gleaning bats, Macroderma gigas and Nyctophilus gouldi. J Comp Physiol A 162:653-668.

Hackett TD, Korine C, Holderied MW (2014): A whispering bat that screams: bimodal switch of foraging guild from gleaning to aerial hawking in the desert long-eared bat. J Exp Biol 217:3028-3032.

Håkansson J, Jakobsen L, Hedenström A, Johansson LC (2017): Body lift, drag and power are relatively higher in large-eared than in smalleared bat species. J R Soc Int 14:20170455.

Holderied M, Korine C, Moritz T (2011): Hemprich's long-eared bat (Otonycteris hemprichii) as a predator of scorpions: whispering echolocation, passive gleaning and prey selection. J Comp Physiol A 197:425-433.

Hopp BH, Arvidson RS, Adams ME, Razak KA (2017): Arizona bark scorpion venom resistance in the pallid bat, Antrozous pallidus. PLoS One 12:e0183215.

Jen PHS, Schlegel PA (1982): Auditory physiological properties of the neurones in the inferior colliculus of the big brown bat, Eptesicus fuscus. J Comp Physiol A 147:351-363.

Kawahara AY, Barber JR (2015): Tempo and mode of antibat ultrasound production and sonar jamming in the diverse hawkmoth radiation. Proc Natl Acad Sci 112:6407-6412.

King AJ, Bajo VM, Bizley JK, Campbell RA, Nodal FR, Schulz AL, Schnupp JW (2007): Physiological and behavioral studies of spatial coding in the auditory cortex. Hear Res 229:106115 .
King AJ, Hutchings ME (1987): Spatial response properties of acoustically responsive neurons in the superior colliculus of the ferret: a map of auditory space. J Neurophysiol 57:596-624

Korine C, Pinshow B (2004): Guild structure, foraging space use, and distribution in a community of insectivorous bats in the Negev Desert. J Zool 262:187-196.

Knudsen EI (1982): Auditory and visual maps of space in the optic tectum of the owl. J Neurosci 2:1177-1194.

Knudsen EI, Blasdel GG, Konishi M (1979): Sound localization by the barn owl (Tyto alba) measured with the search coil technique. J Comp Physiol A 133:1-11.

Kössl M, Hechavarria J, Voss C, Schaefer M, Vater M (2015): Bat auditory cortex - model for general mammalian auditory computation or special design solution for active time perception? Eur J Neurosci 41:518-532.

Macías S, Hechavarría JC, Cobo A, Mora EC (2014): Narrow sound pressure level tuning in the auditory cortex of the bats Molossus molossus and Macrotus waterhousii. Hear Res 309:36-43.

Measor KR, Leavell BC, Brewton DH, Rumschlag J, Barber JR, Razak KA (2017): Matched behavioral and neural adaptations for low sound level echolocation in a gleaning bat, Antrozous pallidus. eNeuro 4:0018.

Moss CF, Sinha SR (2003): Neurobiology of echolocation in bats. Curr Opin Neurobiol 13: 751-758.

Moss CF, Surlykke (2010): Probing the natural scene by echolocation in bats. Front Behav Neurosci 4:33.

Neuweiler G (1990): Auditory adaptations for prey capture in echolocating bats. Physiol Rev 70:615-641.

Neuweiler G (2003): Evolutionary aspects of bat echolocation. J Comp Physiol A 189:245-256.

Neuweiler G, Singh S, Sripathi K (1984): Audiograms of a South Indian bat community. J Comp Physiol A 154:133-142.

Nodal FR, Bajo VM, Parsons CH, Schnupp JW, King AJ (2008): Sound localization behavior in ferrets: comparison of acoustic orientation and approach to target responses. Neuroscience 154:397-408.

Norberg UM, Rayner JM (1987): Ecological morphology and flight in bats (Mammalia; Chiroptera): wing adaptations, flight performance, foraging strategy and echolocation. Phil Trans R Soc Lond B 316:335-427.

Obrist MK, Fenton MB, Eger JL, Schlegel PA (1993): What ears do for bats: a comparative study of pinna sound pressure transformation in Chiroptera. J Exp Biol 180:119-152.

O'Neill WE (1985): Responses to pure tones and linear FM components of the CF-FM biosonar signal by single units in the inferior colliculus of the mustached bat. J Comp Physiol A 157:797-815. 
Razak KA (2011): Systematic representation of sound locations in the primary auditory cortex. J Neurosci 31:13848-13859.

Razak KA (2012): Mechanisms underlying azimuth selectivity in the auditory cortex of the pallid bat. Hearing Res 290:1-12.

Razak KA (2016): Functional segregation of monaural and binaural selectivity in the pallid bat auditory cortex. Hear Res 337:35-45.

Razak KA, Fuzessery ZM (2002): Functional organization of the pallid bat auditory cortex: emphasis on binaural organization. J Neurophysiol 87:72-86.

Razak KA, Fuzessery ZM (2006): Neural mechanisms underlying selectivity for the rate and direction of frequency-modulated sweeps in the auditory cortex of the pallid bat. J Neurophysiol 96:1303-1319.

Razak KA, Fuzessery ZM (2008): Facilitatory mechanisms underlying selectivity for frequency modulated sweeps in the auditory cortex. J Neurosci 28:9806-9816.

Razak KA, Richardson MD, Fuzessery ZM (2008): Experience is required for the maintenance and refinement of FM sweep selectivity in the developing auditory cortex. Proc Natl Acad Sci USA 105:4465-4470.

Razak KA, Shen W, Zumsteg T, Fuzessery ZM (2007): Parallel thalamocortical pathways for echolocation and passive sound localization in a gleaning bat, Antrozous pallidus. J Comp Neurol 500:322-338.

Razak KA, Yarrow S, Brewton D (2015): Mechanisms of sound localization in two functionally distinct regions of the auditory cortex. J Neurosci 35:16105-16115.
Razak KA, Zumsteg T, Fuzessery ZM (2009): Development of auditory thalamocortical connections in the pallid bat, Antrozous pallidus. J Comp Neurol 515:231-242.

Roeder KD (1962): The behaviour of free flying moths in the presence of artificial ultrasonic pulses. Anim Behav 10:300-304.

Roeder KD (1967): Nerve Cells and Insect Behavior. Cambridge, Harvard University Press.

Rübsamen R, Neuweiler G, Sripathi K (1988): Comparative collicular tonotopy in two bat species adapted to movement detection, Hipposideros speoris and Megaderma lyra. J Comp Physiol A 163:271-285.

Russo D, Jones G, Arlettaz R (2007): Echolocation and passive listening by foraging mouseeared bats Myotis myotis and M. blythii. J Exp Biol 210:166-176.

Schnitzler HU, Moss C, Denzinger A (2003): From spatial orientation to food acquisition in echolocating bats. Trends Ecol Evol 18: 386-394.

Simmons JA, Howell DJ, Suga N (1975): Information content of bat sonar echoes: recent research on echolocation in bats identifies some of the kinds of information conveyed by echoes of their sonar sounds. Am Sci 63:204215.

Stecker GC, Harrington IA, Macpherson EA, Middlebrooks JC (2005): Spatial sensitivity in the dorsal zone (area DZ) of cat auditory cortex. J Neurophysiol 94:1267-1280.

Stecker GC, Middlebrooks JC (2003): Distributed coding of sound locations in the auditory cortex. Biol Cybern 89:341-349.
Suga N (1965): Analysis of frequency-modulated sounds by auditory neurones of echo-locating bats. J Physiol 179:26-53.

Suga N (1969): Classification of inferior collicular neurones of bats in terms of responses to pure tones, frequency-modulated sounds and noise bursts. J Physiol 200:555-574.

Suga N (1977): Amplitude spectrum representation in the Doppler-shifted-CF processing area of the auditory cortex of the mustache bat. Science 196:64-67.

Suga N (1984): Neural mechanisms of complexsound processing for echolocation. Trends Neurosci 7:20-27.

Suga N (1989): Principles of auditory information-processing derived from neuroethology. J Exp Biol 146:277-286.

Suga N (1990): Cortical computational maps for auditory imaging. Neural Networks 3:3-21.

Surlykke A, Kalko EK (2008): Echolocating bats cry out loud to detect their prey. PLoS One 3:e2036.

ter Hofstede HM, Schöneich S, Robillard T, Hedwig B (2015): Evolution of a communication system by sensory exploitation of startle behavior. Curr Biol 25:3245-3252.

Waters DA, Jones G (1995): Echolocation call structure and intensity in five species of insectivorous bats. J Exp Biol 198:475-489.

Witzke P (1987): Acuity of pure tone lateralization by the Indian false Vampire bat, Megaderma lyra (Michrochiroptera, Megadermitidae); in Hanak V, Horacek I, Gaisler J (eds): European Bat Research. Praha, Charles University Press, pp 281-286. 